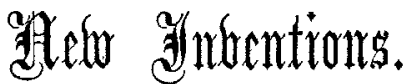

\section{AN ADJUSTABLE HEAD BANDAGE.}

THIS bandage has been invented to meet a need in / size and the extreme rapidity with which it can be applied military as well as hospital and private practice. It is an inexpensive article, and by its use a great saving of time and money will be effected, the final result being equal, in both efficiency and appearance, to the most elaborate bandaging yet used to retain a dressing on the head. Ordinarily many yards of bandage are used each time a complete head dressing is put on, and this material is wasted usually when once removed; in addition, it takes at least five minutes to apply. and pressure on the top of the head is very difficult to obtain. The bandage illustrated can be applied easily in 30 seconds, removed instantly, and reapplied as often as are points of vital importance on the field of battle, where it is possible by its use to bandage the heads of 10 patients. while but one could be done in the former way. In hospitals a great saving of time and materials can be obtained, and in first-aid equipments and the medical man's emergency bag it has a distinct place. The dressing can be removed and reapplied very quickly and neatly, an obvious advantage to the medical man. The bandage may be compared to a skull-cap reaching from the eye-brows to the nape of the neck, thus enclosing the greater part of the head, making it very difficult to dislodge. The side is cut up
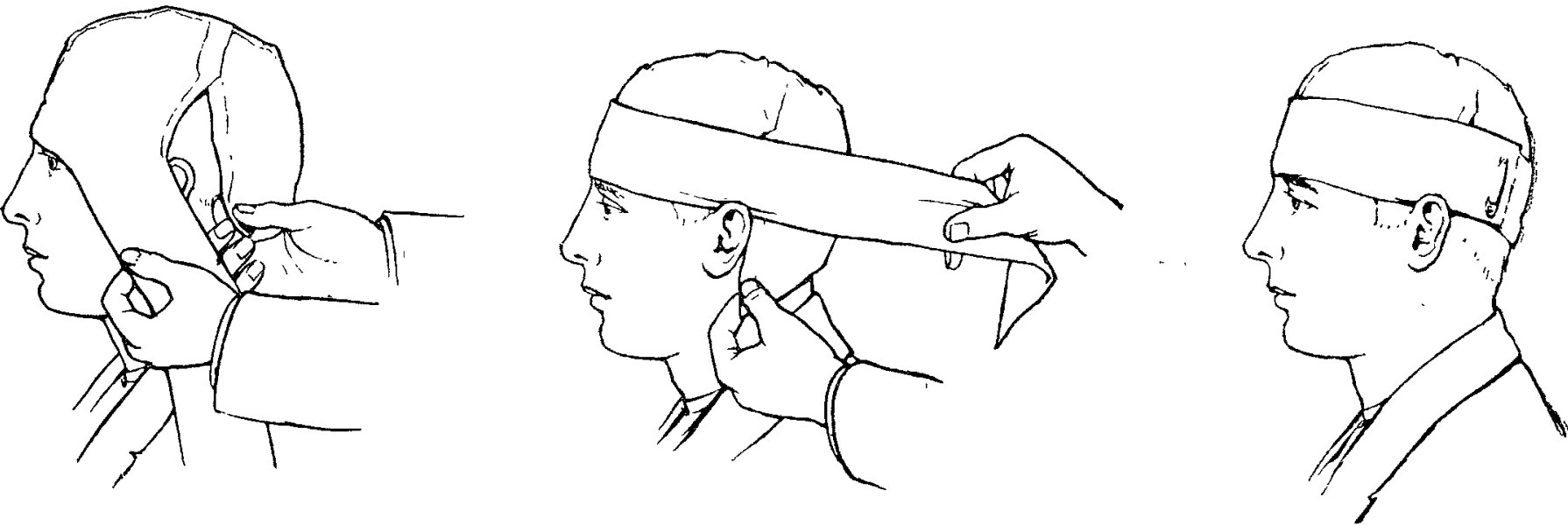

necessary. The pressure can be altered at will, and when the bandage is soiled it can be washed and sterilised an indefinite number of times. No special skill or experience is necessary to apply it. It will fit any head, owing to one side being cut with a gore. The whole article is manufactured from one piece of cloth, reinforced over the top with fine strong tape, so that the bandage is very durable. For military practice a safety-pin, a sterile cyanide gauze dressing, and the bandage are enclosed, compressed, and an impervious cover put over all. The size is $2 \frac{1}{2} \times 1 \frac{1}{1} \times \frac{5}{8}$ in. $=1.9$ cubic inches, or 31.25 c.c., the weight being slightly over $\frac{1}{2}$ oz. The material is of linen, very strong. Its small from the part above the ear to near the top of the head. One lower edge is continued into a bandage which goes a little over the circumference of the head, being $2 \frac{1}{2}$ in. wide. Thus, when lying down, the juncture and pinning positions come at the side, making it very comfortable. After an operation such as trephining a dressing can be very quickly fixed. It has already been tested successfully in actual hospital work.

The bandage can be obtained from Messrs. Burroughs, Wellcome, and Co., Snow Hill Buildings, London, E.C. Gordon G. Copeland, M.B. Toronto.

Toronto, Canada.

\section{TUBE FOR IRRIGATION OF THE AURAL CAVITIES.}

THE tube here figured I have found useful in some cases of suppurative otitis in which there were cholesteatomatous masses in the antrum. I believe that by means of otectomy and irrigation the aural carities can be disinfected, but some cases of cholesteatoma have hitherto been amongst the exceptions, and it is taught in various works of otology that in such cases a radical operation is advisable. Recently I have succeeded in irrigating the antrum by means of my curved tube. This can be intronuced after otectomy, and when in its proper position the opening in the side of the

tube should be opposite the entrance to the antrum (the position of the tube is shown by the indicator), and the irrigating fluid can be sent straight into that cavity. In
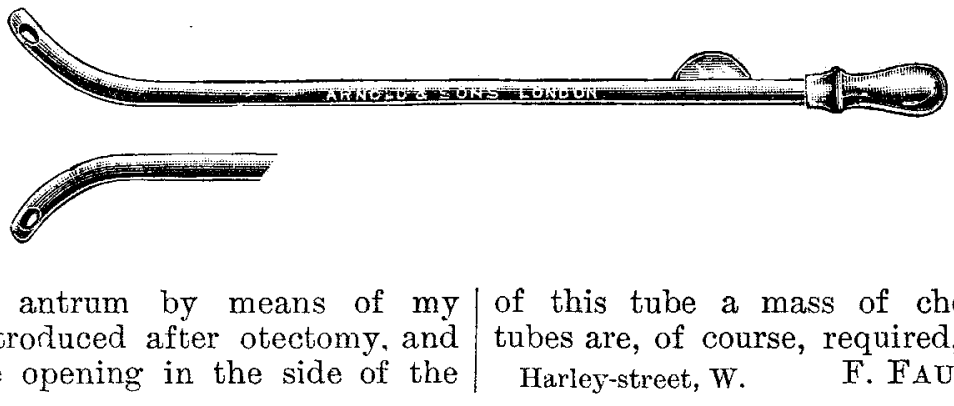
most cases of cholesteatomatous accumulation in the antrum I believe the opening; into that cavity is of considerable size. In one of my cases the offensive odour did not entirely pass away until I had washed out of the antrum by means of this tube a mass of cholesteatomatous matter. Two tubes are, of course, required, one for each side.

Harley-street, W. F. FAULDER WHITE, F.R.C.S. Eng.

Wallasey Hospital Amalgamation: THE Nitional Insurayce ACt. - A joint meeting of the sub. scribers to the Liscard Victoria Central Hospital and the Wallasey Dispensary was held in the board room of the former institution on Nov. 29th, when a resolution was unanimously adopter approving of the amalgamation of the two institutions, which will from Jan. 1st next be known as "The Victoria Central Hospital and Wallasey Dispensary." The invested funds of the dispensary are to be handed over to the committee of the hospital, but to be ear-marked and available only for dispensary work. The chairman said that, in the matter of the Insurance Act, some two or three months ago their medical staff notified them that after Jan. 15th they would no longer agree to attend oratuitously to patients in that hospital who were entitled to benefits under the Act. That raised a new situation. No one found fault with the decision the doctors had come to, but the hospital had no funds to pay its medical staff, and therefore they had to consider the situation. The subcommittee came to the conclusion that if the Act was going to provide money for the relief of persons suffering from sickness or accident, not only were the medical men who attencled them at the hospital entitled to some financial consideration, but the hospital was also entitled to something for their maintenance. The committee decided to notify the Insurance Commissioners to that effect, and there the matter stood. 